\title{
Automatic Sunspots Detection on SODISM Solar Images
}

\author{
${ }^{1}$ Amro F. Alasta, ${ }^{2}$ Abdulrazag Algamudi ${ }^{3}$ Rami Qahwaji ${ }^{4}$ Stanley Ipson and ${ }^{5}$ Tarek A. Nagem \\ 1,2,3,4 and 5 School of Electrical Engineering and Computer Science, University of Bradford
}

\begin{abstract}
The surface of the sun often shows visible sunspots which are located in magnetically active regions of the Sun, and whose number is an indicator of the Sun's magnetic activity. The detection and classification of sunspots are useful techniques in the monitoring and prediction of solar activity. The automated detection of sunspots from digital images is complicated by their irregularities in shape and variable contrast and intensity compared with their surrounding area. The main aim of this paper is to detect sunspots using images from the Solar Diameter Imager and Surface Mapper (SODISM) on the PICARD satellite and calculate their filling factors. A comparison over time with sunspot numbers obtained using images from the SOHO satellite is also presented.
\end{abstract}

Keywords Sunspots; SODISM; Active region; Segmentio, Filling factors.

\section{INTRODUCTION}

The PICARD satellite was launched on 15 June 2010 to observe solar activity using the Solar Diameter Imager and Surface Mapper (SODISM) which provides wide-field images of the photosphere and chromosphere of the Sun in five narrow pass bands (centered at 215.0, 393.37, 535.7, 607.1, and $782.2 \mathrm{~nm}$ ) [1].

The function of SODISM is to continuously monitor solar activity [2], therefore it provides images that can also feed models estimating Solar Spectral Irradiance (SSI) which is relevant to global warming predictions. Moreover, SODISM encompasses the probing of the interior of the Sun via Helioseismic analysis of observations in intensity on the solar disc and at the limb [3].

Figure 1; shows some samples of SODISM images taken early in the mission in all the different pass bands; however, this paper applies only to band $535 \mathrm{~nm}$ images which are the only bands available at level $1 \mathrm{~B}^{1}$ (level $1 \mathrm{~B}$ data products include a number of corrections for instrument issues). The total number downloaded was 206. The format of these image files is FITS and each image has a size of $2048 \times 2048$

\footnotetext{
${ }^{1}$ http://picard.projet.latmos.ipsl.fr/files.php
}

pixels. The obtained data is from 5th August 2010 to 4th January 2014.

The automated detection of solar features is a technique which is widely used for images from ground based observatories and from satellites such as SOHO and SDO but has been relatively underused for images from SODSIM, hence our interest in working with the latter images.

Our main contributions in this paper are:

- Produce a new automated method to detect sunspots from SODSIM images.

- Use Kuwahara and À-Trous filters to remove noise and unwanted dark linear features from images.

- $\quad$ Detect sunspots over the lifetime of SODISM.

- $\quad$ Provide a comparison with images from SOHO.

This paper is arranged as follows. Section 2 summarises some related work, Section 3 describes the Pre-processing methods applied, Section 4 shows the filling factor computations (calculations of the fractions of solar disk regions occupied by sunspots) and Section 5 presents the results. Some conclusions are presented in Section 6.

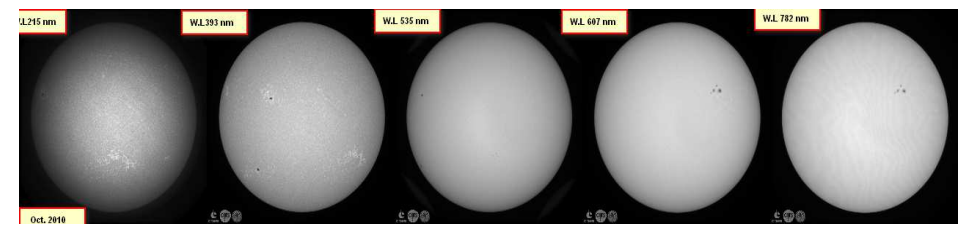

Figure 1: Five Level 1B data product solar images taken on October 2010.

\section{RELATED WORK}

Before segmentation of features on the solar disk can be started it is necessary to determine the boundary of the solar disk (and hence determine its solar radius and center); then we can analyze the interior features. On the solar disk the solar features have a complex distribution of shapes over widely ranging intensities and with no regular patterns. 
Sunspots, the features of interest here, are dark, sometimes irregularly shaped, local structures on the solar disk. The automatic sunspots segmentation system outlined here requires no supervision; no previous parameters and no heuristics to perform the stages of segmentation. There are three basic types of segmentation methods:

- Boundary based.

- Region based.

- Thresholding approaches.

Determining the boundaries between regions is done by looking for discontinuities in gray levels as in Zharkov et al. [4] where Gauss smoothing and Sobel gradient are applied to detect contours, using operators sensitive to meaningful discontinuities in intensity level. However, in most applications of this process, especially to complex structures, the results will not be satisfactory, so post-processing operations are required. The review made by Zharkov et al. [5] evaluated and summarized the existing manual, semi-automated and fully automated feature recognition techniques applied to different solar features including sunspots and networks.

An example of a region based approach is the Watershed method, which produces catchment basins between ridges corresponding to minimum of gradients, often leading to over segmentation. Thus, again, a complex selection of markers and additional modifications are necessary [6].

The simplest and quickest method to apply is thresholding [7]. However, global thresholding of the solar disk is not a practical solution because of the non-uniform brightness of the background solar disk caused by factors such as the limb darkening effect due to varying absorption of light in different thicknesses of the solar atmosphere traversed by rays from the sun to the detector. However, this can be corrected by normalization of the image brightness in a pre-processing step [8]. Moreover, some background regions of the solar disk in some images could be darker than some sunspots in other regions.

A method for the detection of sunspots from SODISM images (band $535 \mathrm{~nm}$ from 5th August 2010 to 4th January 2014) is introduced in internal work ${ }^{2}$ [9], Wavelet Harr filter was applied to remove noise from the image, followed by band pass filter to remove limb darkening. Gaussian smoothing was applied next to remove isolated noisy pixels. The correlation coefficient between SOHO and SODSIM images was found to be 0.98 .

2 https://projects.pmodwrc.ch/solid/index.php/links/10-news-archive/31deliverables
In 2008, Curto et al. [7] Developed fully automated recognition method for sunspots (dark features) using morphological operators.

in $2016 \mathrm{M}$. Meftah and et al. [2] working on SODISM $393 \mathrm{~nm}$ data to detect sunspots and faculae (bright features) applied a similar method to Corto et al. using morphological operations Their method has the following steps:

- $\quad$ Preprocessing steps are applied to SODISM images (L1 data) in order to obtain images with full contrast. Median filtering is applied to reduce noise.

- For sunspot detection, morphological processing consisting of a top-hat operation is applied to the preprocessed images.

- Segmentation of sunspots is performed using an Otsu threshold. However, their method gives better results if manual thresholding is used.

- For faculae detection, morphological processing consisting of a bottom-hat (complement of the tophat) operation is performed.

- An automated threshold is applied to the image, in order to detect faculae but if the number of detected faculae is not coherent, an iterative procedure is launched, starting from a reduced threshold and/or increasing it gradually until the number of detected faculae belongs to a fixed interval, These steps are the most time consuming.

An example of a processed $393.37 \mathrm{~nm}$ SODSIM image of Meftah method is shown in Figure 2.

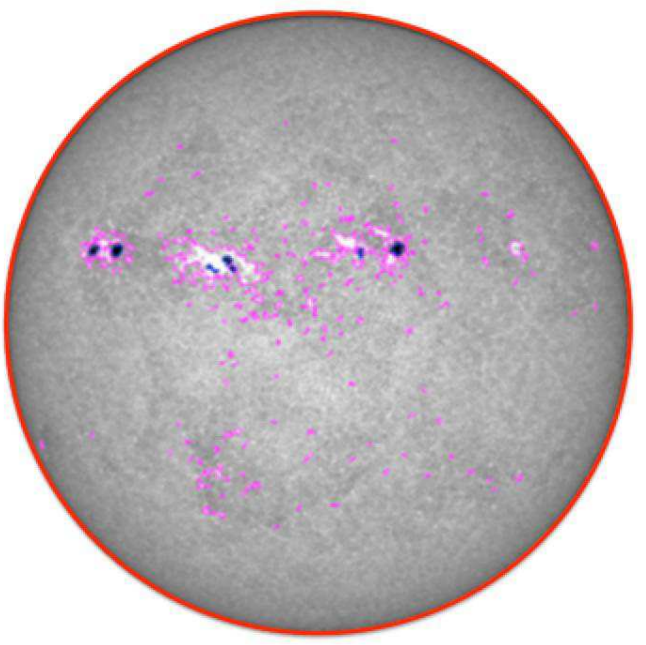

Figure 2: Example of solar disc segmentation for a $393.37 \mathrm{~nm}$ SODISM image. Red marks the solar boundary, blue marks the detected sunspots and 
magenta marks the detected faculae. The quality of the detection is governed by thresholds used[2].

\section{PRE-PROCESSING AND FEATURES DETECTION}

The previous methods for detecting sunspots with SODISM images have various limitations; which include manually adjusted thresholds, time consuming operation, application to $393 \mathrm{~nm}$ rather than $535 \mathrm{~nm}$ images and difficulty working with huge amounts of data.

Our method overcomes the problems of working with lots of data because it is fully automated. It was applied on $535 \mathrm{~nm}$ images but not tested $393 \mathrm{~nm}$ images because these were not available in 1B form.

The method developed to automatically detect sunspots in $535.7 \mathrm{~nm}$ SODISM L1 images and programmed in MATLAB includes the following steps.

- First detect the solar disk and record its centre and radius information and eliminate all information located outside the solar disk.

- Convert the image scale from signed 32 bit to unsigned 8 bit, in preparation for application of Kuwahara and A trous filters in the next step.

- Remove noise and some other unwanted features from images by applying a combination of Wavelet based Kuwahara and A trous filters. The former is a non-linear smoothing filter with good noise-reduction properties at the same time preserving edges so it is used for adaptive noise reduction $[10]^{3}$. The second filter is an À-Trous noise reduction filter which makes use of image reconstruction from thresholded 'A trous' wavelet transform coefficients. Linear dark features found in some images, which would otherwise be detected as sunspots, are also removed by this step Thresholding factors are specified to control the amount of noise removed

- $\quad$ Correct any pixels which are brightness outliers.

- Apply a Band pass filter to display sunspots on a normalized background.

- Threshold to obtain a mask image determining the sunspot locations.

Examples of an input solar image and the corresponding detected sunspots are shown in Figure 3.

3 https://imagej.nih.gov/ij/plugins/kuwahara.html
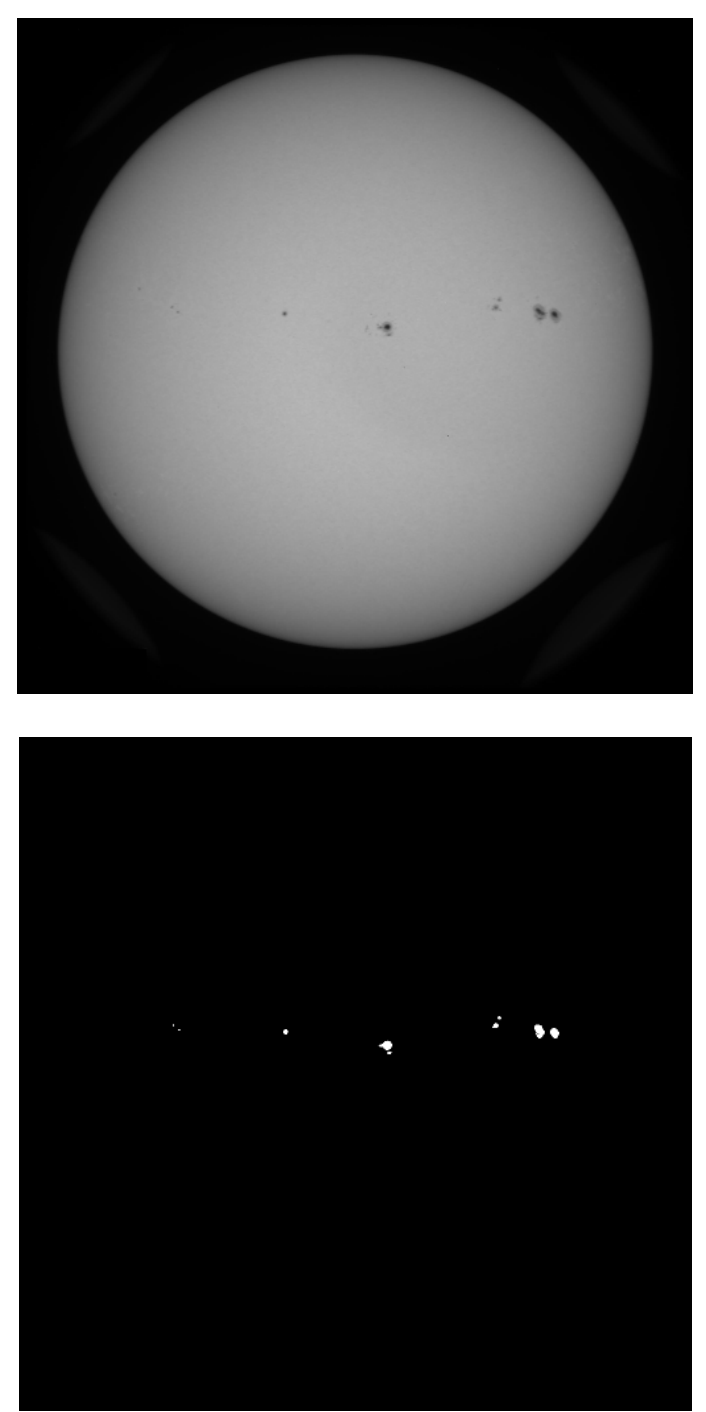

Figure 3: A SODISM 535 image from 12:30, $1^{\text {th }}$ October 2011 (top) and resulting detected sunspots (bottom).

\section{FILLING FACTORS COMPUTATION}

The calculated filling factors for a feature reflect the fraction of the solar disk covered by the feature and the assignment of reference synthetic spectra to it has applications to modelling the Solar Spectral Irradiance [11]. The filling factor is calculated as a function of radial position on the sun disk. The solar disk is divided by 11 concentric rings starting with an inner radius RI and an outer radius RO. In tables 1 and 2 these radii are defined as ratios relative to the radius of the solar disc. In the case of SODISM images the last interval would be between 0.95 and the boundary of the solar disk. All sunspot pixels which are crossed by one of the concentric rings have their areas appropriately 
apportioned between the two adjacent regions. A consequence of this can be seen in rows 8 and 9 of Table 2, which shows an example of sunspot filling factors calculated for the 5th August 2010, 04:49.

TABLE 1. RELATIVE RADIUS VALUES.

\begin{tabular}{|c|c|c|}
\hline Index & $\begin{array}{c}\text { Inner radius } \\
\text { (relative radius) }\end{array}$ & $\begin{array}{c}\text { Outer radius } \\
\text { (relative radius) }\end{array}$ \\
\hline 1 & 0.00 & 0.07 \\
\hline 2 & 0.07 & 0.16 \\
\hline 3 & 0.16 & 0.25 \\
\hline 4 & 0.25 & 0.35 \\
\hline 5 & 0.35 & 0.45 \\
\hline 6 & 0.45 & 0.55 \\
\hline 7 & 0.55 & 0.65 \\
\hline 8 & 0.65 & 0.75 \\
\hline 9 & 0.75 & 0.85 \\
\hline 10 & 0.85 & 0.95 \\
\hline 11 & 0.95 & 1.05 \\
\hline
\end{tabular}

TABLE 2. NUMBERS OF SUNSPOT IN THE DIFFERENT PARTS OF THE SOLAR DISK ON 5TH AUGUST 2010, 04:49.

\begin{tabular}{|c|c|c|c|}
\hline Index & Inner radius & Outer radius & Sunspots \\
\hline 1 & 0 & 0.07 & 0 \\
\hline 2 & 0.07 & 0.16 & 0 \\
\hline 3 & 0.16 & 0.25 & 0 \\
\hline 4 & 0.25 & 0.35 & 0 \\
\hline 5 & 0.35 & 0.45 & 1170 \\
\hline 6 & 0.45 & 0.55 & 0 \\
\hline 7 & 0.55 & 0.65 & 0 \\
\hline 8 & 0.65 & 0.75 & 18.55 \\
\hline 9 & 0.75 & 0.85 & 94.45 \\
\hline 10 & 0.85 & 0.95 & 338 \\
\hline 11 & 0.95 & 1.05 & 0 \\
\hline \multicolumn{2}{|l|}{ Total of Pixels } & & 1621 \\
\hline
\end{tabular}

\section{RESULTS}

The segmentation process outlined earlier has been applied to the entire downloaded $535 \mathrm{~nm}$ image data, in order to detect sunspots and calculate their filling factors. Figure 4 shows the results of sunspot filling factor calculations for data from October 2010 until the end of life of the Picard satellite, 1 January 2014. Moreover, in the period from August, 6th 2010 to December 19th 2010, when data from both sources are available, a comparison between sunspots' filling factors calculated for SODISM $535 \mathrm{~nm}$ images and MDI intensitygram images from the SOHO satellite has been done. The most common of comparison method between two values, or measure of dependence between two quantities, is "Pearson's correlation coefficient", commonly called "the correlation coefficient". This is obtained by dividing the covariance of the two variables by the product of their covariance of the two variables by the product of their standard deviations as follows.

$$
\operatorname{Correl}(X, Y)=\frac{\sum(x-\bar{x})(y-\bar{y})}{\sqrt{\sum(x-\bar{x})^{2} \sum(y-\bar{y})^{2}}}
$$

Where $\bar{x}$ and $\bar{y}$ are the means of arrays $x$ and $y$ respectively.

Figure 5 shows the results. The correlation coefficient between the two data sets is 0.985 . This is meaning have a strong positive linear correlation.

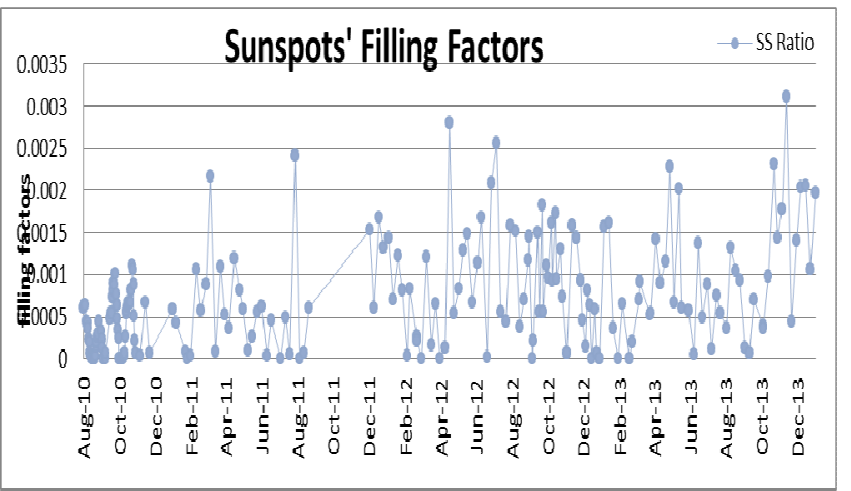

Figure 4: Sunspot filling factors calculated from SODISM 535 images over the lifetime of the satellite.

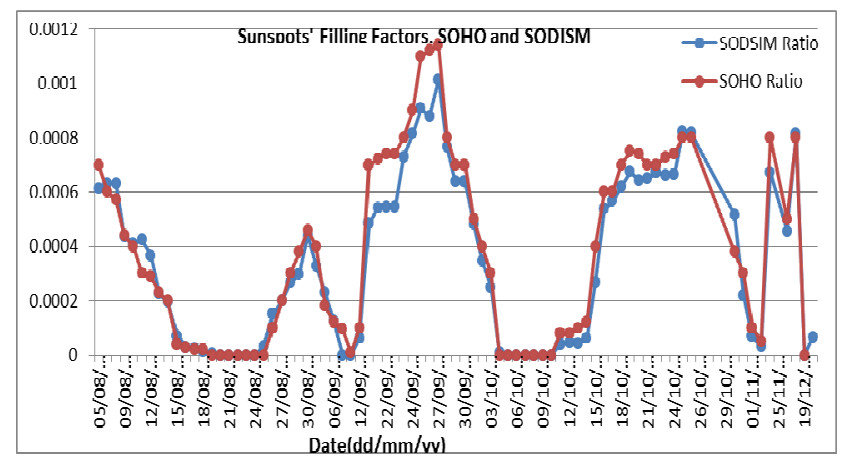

Figure 5: A comparison of sunspot filling factors calculated from SOHO and SODISM images.

\section{CONCLUSIONS}

Despite of decreasing quality of SODSIM images through its lifetime, automatic detection of sunspots in SODISM images is possible with suitable pre-processing procedures. 
The previewed method by M. Meftah et al, applied to 393 $\mathrm{nm}$ SODISM images needs manually interaction to optimize the threshold which is calculated using the Otsu method. This is based on the histogram of all the interior solar disc pixels. Since the thresholds are computed on the differently preprocessed images, the thresholds used to detect sunspots and faculae are different even though the same method is used to compute them both.

The second method, which is used by Qahwaji et al in his internal work [9], achieves good results and a correlation coefficient of 0.98 between SODISM and SOHO images, only slightly less than that achieved in the same period in this work.

The method adopted here successfully detects sunspots on $535 \mathrm{~nm}$ images over the lifetime of the satellite and then calculates their filling factors, Furthermore, the comparison of sunspots' filling factors between SOHO and SODSIM images shows an excellent match over the early period when they are both available, with a correlation coefficient of 0.985 . This is slightly better than the previous method by Qahwaji et al, which was 0.98 [9]. This method here is completely automated which make it easy to apply on massive data images, unlike the Otsu threshold based method mentioned earlier, which needs manual control.

Although the overall correlation is high, inspection of the results in Figure 6 shows the filling factors for SODISM and SOHO are systematically slightly different in amplitude despite mostly changing in step. This is most evident over the period from 22 Sep 2010 to 29 Sep 2010 when there is a somewhat lower correlation coefficient (0.924). This may well be related to the fact that the SOHO data corresponds to a different wavelength $(676.8 \mathrm{~nm})$ to the SODSIM images. However, the results shown in Figure 5 suggest that this method of automatic segmentation can be applied on the $535 \mathrm{~nm}$ data giving useful results despite long term decreasing quality of the SODISM images.

\section{REFERENCES}

A. Hauchecorne, M. Meftah, A. Irbah, S. Couvidat, and R. Bush, "To cite this version: HAL Id: hal-00952209 Solar radius determination from SODISM / PICARD and HMI / SDO observations of the decrease of the spectral solar radiance during the June," vol. 783, no. 2, 2014.

[2] M. Meftah, T. Corbard, A. Hauchecorne, A. Irbah, P. Boumier, A. Chevalier, W. Schmutz, R. Ikhlef, F. Morand, C. Renaud, J.-F. Hochedez, G. Cessateur, S. Turck-Chièze, D. Salabert, M. Rouzé, M. van Ruymbeke, P. Zhu, S. Kholikov, S. Koller, C. Conscience, S. Dewitte, L. Damé, and D. Djafer, "Main results of the PICARD mission,” no. July, p. 99040Z, 2016.

[3] M. Meftah, a Hauchecorne, T. Corbard, E. Bertran, M. Chaigneau, and M. Meissonnier, "PICARD SODISM, a space telescope to study the Sun from the middle ultraviolet to the near infrared," no. January, pp. 1-38, 2014.

[4] V. Zharkova, S. Ipson, A. Benkhalil, and S. Zharkov, "Feature recognition in solar images," Artif. Intell. Rev., vol. 23, no. 3, pp. 209-266, 2005.

[5] S. Zharkov, V. Zharkova, S. Ipson, and A. Benkhalil, "Technique for automated recognition of sunspots on full-disk solar images," EURASIP J. Appl. Signal Processing, vol. 2005, no. 15, pp. 2573-2584, 2005.

[6] E. J. Kacprzyk, "Soft Computing in Industrial Applications," Soft Comput., no. June, p. 438, 2011.

[7] J. J. Curto, M. Blanca, and E. Martínez, "Automatic sunspots detection on full-disk solar images using mathematical morphology," Sol. Phys., vol. 250, no. 2, pp. 411-429, 2008.

[8] S. Zhang, H. Yang, and L. Singh, "Increased information leakage from text," CEUR Workshop Proc., vol. 1225, no. 2003, pp. 4142, 2014.

[9] O. A. Rami Qahwaji, Stan Ipson, "SOLID D3.5 Filling factors catalogue for PICARD images SOLID," First Eur. Compr. Sol. Irradiance Data Exploit., pp. 1-8, 2015.

[10] H. Al-Marzouqi, "Data Clustering Using a Modified Kuwahara Filter," in 2009 International Joint Conference on Neural Networks, 2009, pp. 128-132.

[11] O. Ashamari, R. Qahwaji, S. Ipson, M. Schöll, O. Nibouche, and M. Haberreiter, "Identification of photospheric activity features from SOHO/MDI data using the ASAP tool," J. Sp. Weather Sp. Clim., vol. 5, p. A15, 2015. 